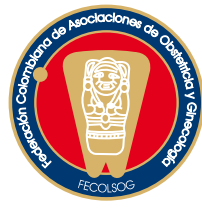

\title{
DOLOR PÉLVICO CRÓNICO EN MUJERES: UNA REVISIÓN SISTEMÁTICA DE ESTUDIOS DE LA PREVALENCIA POBLACIONAL 2004-2009
} A systematic review of population prevalence studies
regarding chronic pelvic pain in females, $2004-2009$ Adalberto Campo-Arias, M.D.*

\section{RESUIMEN}

Introducción: el dolor pélvico crónico (DPC) es un síndrome de alta prevalencia en mujeres de la población general y una de las causas más frecuentes de consulta a los servicios de salud. Sin embargo, no hay disponibles revisiones sistemáticas recientes de la prevalencia de DPC en la comunidad general en los últimos cinco años.

Objetivo: conocer la prevalencia de DPC en mujeres de la población general en estudios publicados entre enero de 2005 y diciembre de 2009.

Materiales y métodos: se realizó una revisión sistemática de artículos que informaron la prevalencia de DPC en mujeres adultas de la población general. Se incluyeron artículos que se publicaron en español, inglés o portugués, entre enero de 2005 y diciembre de 2009. Se excluyeron las revisiones sistemáticas. Se realizó la búsqueda en BIREME, EBSCO, IMBIOMED, LILACS, MEDLINE, Ovid y ProQuest. Los Descriptores en Ciencias de la Salud que se usaron fueron: dolor pélvico (crónico) ("chronic pelvic pain"; "dor pélvica”), mujeres ("women" o "female”; "mulheres"), adulto ("adult"; "adulto"), epidemiología ("epidemiology”; “epidemiologia”) y

* Médico Especialista en Psiquiatría. Maestrante en Salud Sexual y Reproductiva. Grupo de Investigación del Comportamiento Humano, Director de Investigaciones y Publicaciones del Instituto de Investigación del Comportamiento Humano. Bogotá (Colombia). Correo electrónico: campoarias@comportamientohumano.org prevalencia ("prevalence"; "prevalência"). Se realizó un análisis cualitativo de los datos.

Resultados: se encontraron 79 títulos de los cuales se incluyeron cuatro estudios de algunas comunidades de Australia, Estados Unidos, Austria y Nueva Zelanda que encontraron prevalencias de DPC entre 5,7 y 26,5\%.

Conclusiones: la prevalencia de DPC es variable en mujeres. Se necesitan más estudios en población general, en países desarrollados y en vía de desarrollo.

Palabras clave: dolor pélvico crónico, prevalencia, mujeres, adulto, revisión sistemática.

\section{SUIMMARY}

Introduction: chronic pelvic pain (CPP) is a syndrome having high prevalence in females in the general population and is one of the most frequent causes for them to consult health services. However, there have been no recent systematic reviews of $\mathrm{CPP}$ prevalence in the general community during the last five years.

Objective: ascertaining CPP prevalence in females in the general population in studies published between January 2005 and December 2009.

Materials and methods: a systematic review was made of articles reporting CPP prevalence in adult females in the general population. Articles were included which had been published in Spanish, 
English or Portuguese between January 2005 and December 2009. Systematic reviews were excluded. The search was carried out in BIREME, EBSCO, IMBIOMED, LILACS, MEDLINE, Ovid and ProQuest. The Health Sciences Descriptors used were: dolor pélvico (crónico) ("chronic pelvic pain”; “dor pélvica”), mujeres ("women" o "female"; "mulheres"), adulto ("adult"; "adulto"), epidemiología ("epidemiology"; "epidemiologia") and prevalencia ("prevalence"; "prevalência"). A qualitative analysis was made of the data.

Results: 79 titles were found, of which 4 studies were included which dealt with some communities in Australia, the USA, Austria and New Zealand which found $5.7 \%$ to $26.5 \%$ CPP prevalence.

Conclusions: CPP prevalence is variable in females. More studies should be carried out in the general population in both developed and developing countries.

Key words: chronic pelvic pain, prevalence, female, adult, systematic review.

\section{INTRODUCCIÓN}

Se define el dolor pélvico crónico (DPC) como la presencia de dolor en la parte inferior del abdomen que no se relaciona con el ciclo menstrual o la actividad sexual, está presente por lo menos durante seis meses y no se precisa una causa específica o explicación razonable. ${ }^{1-3} \mathrm{El}$ DPC puede presentarse en cualquier área de la zona pélvica y se irradia a diferentes áreas abdominales. En algunos casos, la intensidad y la duración varían según los días del ciclo menstrual, características que dan claves importantes sobre la etiología. ${ }^{4,5}$

El DPC es un síndrome que afecta tanto a hombres como a mujeres. ${ }^{6,7}$ Sin embargo, es más común en mujeres ${ }^{6,7}$ y es una de las causas más frecuentes de consulta a los servicios de salud. ${ }^{4}$ Además, muchas mujeres no consultan o no reciben un diagnóstico apropiado de la condición. ${ }^{8-10}$ El DPC en mujeres es una condición de larga evolución y pobre respuesta a intervenciones farmacológicas y quirúrgicas, ${ }^{11,12}$ con importantes consecuencias a corto y largo plazo como mala calidad de vida, depresión y ansiedad. ${ }^{13-23}$
En relación con la prevalencia de DPC en mujeres, Latthe y sus colegas llevaron a cabo una revisión sistemática de 18 estudios publicados hasta 2004 que informaban sobre la prevalencia de este problema de salud pública en la población general y observaron que la frecuencia global de DPC no cíclico se hallaba entre el 4,0 y el 43,4\%. Asimismo, mostraron que la prevalencia variaba ampliamente en países menos desarrollados como la India, $\mathrm{Pa}$ kistán y Tailandia, con valores entre 4,2 y 42,2\%. No obstante, la prevalencia se redujo cuando se analizaron los estudios de mayor calidad, con muestras representativas, en donde la prevalencia varió entre 2,1 y $29,9 \% .^{24}$

Es importante conocer la prevalencia de DPC con el propósito de tomar medidas desde la perspectiva de la salud pública y, de esta manera, diseñar estrategias para la identificación temprana, mediante un abordaje multidisciplinario de los posibles casos y con ello reducir la carga de enfermedad que representa el DPC y minimizar el deterioro en la calidad de vida de la población femenina. ${ }^{25-32}$

El objetivo de esta revisión sistemática fue conocer la prevalencia de DPC en mujeres de la población general en estudios publicados entre enero de 2005 y diciembre de 2009.

\section{MATERIALES Y MÉTODOS}

Con base en los Descriptores en Ciencias de la Salud (DeCS): “dolor pélvico” (crónico), “mujeres”, "adulto", "epidemiología" y "prevalencia", se realizó una búsqueda en las bases de datos BIREME, EBSCO, Google académico, IMBIOMED, LILACS, Ovid, ProQuest y MEDLINE vía PubMed de artículos originales en los que se indagó sobre la prevalencia de DPC en mujeres adultas de la población general. Se incluyeron artículos que se publicaron en español, inglés o portugués entre enero de 2005 y diciembre de 2009. Para completar la búsqueda se revisaron las referencias que presentaban cada uno de los artículos que cumplieron con los criterios de inclusión. Se excluyeron las revisiones sistemáticas y los metaanálisis. 
Inicialmente, se revisaron los títulos, seguidamente los resúmenes y, por último, los artículos completos que reunían los criterios de inclusión. Se utilizó una ficha que resumió las características de la población, la definición de DPC empleada y la prevalencia. Se realizó un análisis cualitativo de los estudios. Cuando no estuvo disponible, se estimó el intervalo de confianza al 95\% (IC95\%) para la prevalencia de DPC.

\section{RESUILTADOS}

De la búsqueda se obtuvieron 79 títulos, de los cuales se excluyeron 7 revisiones y 67 investigaciones que informaron la prevalencia de otros problemas pélvicos no relacionados con DPC y estudios de intervención. Finalmente, 5 artículos fueron aptos para ingresar a la revisión. Un artículo se excluyó porque investigó la presencia de dolor pélvico como síntoma de infección del tracto reproductivo. ${ }^{33}$ No se encontraron estudios latinoamericanos sobre la prevalencia de DPC en personas de la comunidad general durante el período revisado.

En Estados Unidos, Bachman et al enviaron un cuestionario por correo a 4.872 mujeres a quienes preguntaron sobre la presencia de dolor ginecológico durante los seis últimos meses. A las mujeres que informaron dolor crónico se les preguntó la localización (uterino, ovárico, intravaginal o extravaginal), la naturaleza del dolor (persistente o episódico) y las situaciones que exacerbaban la condición dolorosa. Un total de 1.772 participantes devolvió completo el cuestionario (media para la edad 50,2 años, desviación estándar 10,5); se observó que el 21,3\% informaba dolor ginecológico crónico y $17,1 \%$, dolor ginecológico no vulvar (sugestivo de DPC). ${ }^{34}$

En Nueva Zelanda, Grace y Zondervan enviaron un cuestionario por correo a una muestra aleatoria de 1.160 mujeres de la población general (comunidad), entre 18 y 50 años y definieron DPC como un dolor en la región más baja del abdomen que no se relacionaba con el ciclo menstrual, la actividad sexual o el embarazo durante los tres meses previos. Un total de 1.079 mujeres respondieron el cuestionario (93\% de respuesta). Las autoras informaron que 26,5\% presentaba DPC; 26,5\%, dismenorrea; 10,2\%, dispareunia, y 36,8\%, sin dolor pélvico. ${ }^{35}$

En Australia, Pitts et al entrevistaron a un grupo de 1.983 mujeres, entre 18 y 49 años, sexualmente activas y que presentaron alguna menstruación durante los doce meses previos. Para el estudio definieron DPC como el dolor pélvico que no se presenta durante la menstruación o el coito, constante o intermitente. Los autores informaron que 21,5\% presentaba DPC; 71,7\%, dismenorrea, y 14,1\%, dispareunia (categorías que no eran mutuamente excluyentes). ${ }^{36}$

En Austria, Marszalek et al realizaron un análisis transversal de una cohorte de personas y evaluaron la presencia del síndrome de DPC durante la última semana o el último mes en hombres y mujeres con el Índice de Dolores Prostáticos Crónicos (Chronic Prostatitis Symptom Index) que diseñó el Instituto Nacional de Salud (National Institute of Health). Este cuestionario tuvo las adaptaciones respectivas para la población femenina. Los autores consideraron que los participantes con puntuaciones iguales o superiores a cuatro sugerían DPC. En esta investigación participaron 981 mujeres entre 20 y 79 años de edad (media 49,1 años; $\mathrm{DE}=0,5)$ y $5,7 \%$ presentaban síntomas sugestivos de DPC. Además, los datos se manejaron con estadística paramétrica. ${ }^{37}$

En la tabla 1 se resume la información acerca del número de participantes, la prevalencia de DPC con su respectivo intervalo de confianza del 95\% (IC95\%).

En relación con la calidad de los estudios, es importante anotar que la prevalencia se estimó a partir de análisis transversal con muestras con distintos criterios de inclusión y exclusión, en particular la edad. Tres estudios cuantificaron los síntomas mediante el uso de instrumentos autoaplicados y un estudio mediante entrevista 
Tabla 1. Prevalencia de DPC en los estudios incluidos.

\begin{tabular}{|c|c|c|c|}
\hline Autores & Muestra & Frecuencia & Prevalencia (IC95\%) \\
\hline Bachman et $a l^{34}$ & 1.772 & 303 & $17,1(15,3-18,9)$ \\
\hline Grace y Zondervan ${ }^{35}$ & 1.079 & 286 & $26,5(23,9-29,1)$ \\
\hline Pitts et $a l^{36}$ & 1.983 & 427 & $21,5(19,7-23,3)$ \\
\hline Marszalek et $a l^{37}$ & 981 & 56 & $5,7(4,2-7,2)$ \\
\hline
\end{tabular}

clínica. Se observaron diferencias en la forma como se realizó la definición de DPC en relación con la duración del dolor, entre una semana o un mes hasta seis meses como tiempo. Asimismo, se desconocía la validez y confiabilidad de algunos cuestionarios que se emplearon; por ejemplo, el Índice de Dolores Prostáticos Crónicos del Instituto Nacional de Salud que usaron Marszalek et al se diseñó para el uso en hombres y no contaba con un proceso de validación formal en mujeres al momento de la aplicación. ${ }^{37}$

\section{DISCUISIÓN}

En el presente estudio se encontró una amplia variación en la prevalencia de síntomas sugestivos de DPC en mujeres, con valores entre 5,7 y 26,5\%. Sin embargo, Latthe et al, en estudios publicados hasta 2004, observaron un rango de prevalencia mayor, entre el 4,0 y el 43,4\%, sin considerar la calidad de los estudios. ${ }^{24}$

Sin duda, es posible que las diferencias en las prevalencias y variables asociadas a DPC que se observaron entre los estudios revisados y las revisiones sistemáticas precedentes se puedan explicar por la variedad de instrumentos y los criterios y las estrategias que usaron para definir DPC, y las características demográficas de las mujeres participantes. ${ }^{38}$ Es necesario el diseño de estudios epidemiológicos que controlen los posibles errores y sesgos en estudios poblacionales. $^{39,40}$

No se encontraron estudios que informen claramente la prevalencia de DPC en mujeres de la población general de Colombia y Suramérica. Los estudios nacionales del dolor no estimaron la prevalencia de DPC con los criterios que antes se citaron, por ello no se incluyeron en esta revisión. ${ }^{41-45}$ Es importante conocer la prevalencia de DPC en mujeres colombianas y en otros países de todos los continentes mediante el diseño de estudios epidemiológicos que superen las limitaciones de las investigaciones disponibles, por ejemplo, una definición uniforme para DPC que permita comparaciones por países o regiones. ${ }^{6}$ Asimismo, es necesario fortalecer la formación de los futuros médicos en el abordaje diagnóstico inicial en el contexto clínico, que incluya una evaluación cuidadosa de los aspectos físicos y emocionales de las mujeres con DPC. ${ }^{46-48}$

La fortaleza que esta revisión sistemática presenta es que resume las investigaciones en población general sobre DPC en los últimos cinco años. La búsqueda incluyó artículos en español y portugués habitualmente omitidos. Sin embargo, cuenta con algunas limitaciones como incluir un número pequeño de investigaciones de baja calidad, heterogéneas, con definiciones distintas para DPC y poblaciones no representativas. No obstante, estas limitaciones son similares a las que se encontraron en revisiones antes del año 2004. ${ }^{24,49,50}$

\section{CONCLUISIONES}

La prevalencia de síntomas sugestivos de DPC es variable en la población general de las mujeres en algunas comunidades de Australia, Estados Unidos, Austria y Nueva Zelanda. En todo el mundo, se necesitan estudios epidemiológicos bien diseñados para determinar la prevalencia de DPC. 


\section{REFERENCIAS}

1. Gómez PI, Silvestre P, Bonilla M, Cayuela C, Medina A, Férnandez S, et al. Dolor pélvico crónico en la mujer. Rev Colomb Obstet Ginecol 1994;45:27-32.

2. Duffy S. Chronic pelvic pain: defining the scope of the problem. Int J Gynaecol Obstet 2001;74:S3-7.

3. Vercellini P, Somigliana E, Viganò P, Abbiati A, Barbara G, Fedele L. Chronic pelvic pain in women: etiology, pathogenesis and diagnostic approach. Gynecol Endocrinol 2009;25:149-58.

4. Gómez PI, Castillo DT. Dolor pélvico. En: Hernández JJ, Moreno C (eds). Medicina del dolor. Bogotá: Ediciones Rosaristas; 2005. p. 228-42.

5. Neis KJ, Neis F. Chronic pelvic pain: cause, diagnosis and therapy from a gynaecologist's and an endoscopist's point of view. Gynecol Endocrinol 2009;25:757-61.

6. Zondervan K, Kennedy SH. Epidemiology of chronic pelvic pain. Int Cong Ser 2005;1279:77-84.

7. Pitts M, Ferris J, Smith A, Shelley J, Richters J. Prevalence and correlates of three types of pelvic pain in a nationally representative sample of Australian men. J Sex Med 2008;5:1223-9.

8. Zondervan KT, Yudkin PL, Vessey MP, Jenkinson CP, Dawes MG, Barlow DH, et al. Chronic pelvic pain the community-symptoms, investigations, and diagnoses. Am J Obstet Gynecol 2001;184:1149-55.

9. Grace VM, Zondervan KT. Chronic pelvic pain in New Zealand: prevalence, pain severity, diagnoses and use of the health services. Aust N Z J Public Health 2004;28:369-75.

10. Hernández JJ. El dolor en la mujer - Dolores reales, mujeres reales. En: Hernández JJ, Gómez PI (eds). Dolor en la Mujer. Asociación Colombiana para Estudio del Dolor (ACED); 2008. p. 15-36.

11. Lamvu G, Williams R, Zolnoun D, Wechter ME, Shortliffe A, Fulton G, et al. Long-term outcomes after surgical and nonsurgical management of chronic pelvic pain: one year after evaluation in pelvic pain specialty clinic. Am J Obstet Gynecol 2006;195:591-600.

12. Weijenborg PT, Greeven A, Dekker FW, Peters AA, Ter Kuile MM. Clinical course of chronic pelvic pain in women. Pain 2007;132:S117-23.

13. Garcés H. Dolor pélvico crónico y laparoscopia. Profamilia. Medellín. Julio-septiembre 1989. Rev CES Med 1990;4:91-5.

14. Silvestre P, Gómez P, Bonilla M, Cayuela C, Medina A, Fernández S, et al. Aspectos psicosociales de las mujeres con dolor pélvico crónico que consultan a la clínica de dolor del Instituto Materno Infantil. Rev Colomb Obstet Ginecol 1993;44:303-6.

15. Reed BD, Haefner HK, Punch MR, Roth RS, Gorenflo DW, Gillespie BW. Psychosocial and sexual functioning in women with vulvodynia and chronic pelvic pain. A comparative evaluation. J Reprod Med 2000;45:624-32.

16. Criollo CP, Romero NM, Salazar OL, Briceño F, Navarro H, Herrera JA. Características ginecológicas y psicosociales del dolor pélvico crónico. Rev Colomb Obstet Ginecol 2004;55:129-35.

17. Smith BH, Elliot AM, Chambers WA, Smith WC, Hannaford PC, Penny K. The impact of chronic pain in the community. Fam Pract 2001;18:292-9.

18. Roth RS, Punch MR, Bachman JE. Educational achievement and pain disability among women with chronic pelvic pain. J Psychosom Res 2001;51:563-9.

19. Leserman J, Zolnoun D, Meltzer-Brody L, Lamvu G, Steege JF. Identification of diagnostic subtypes of chronic pelvic pain and how subtypes differ in health status and trauma history. Am J Obstet Gynecol 2006;195:554-60.

20. Lorençatto C, Petta CA, Navarro MJ, Bahamondes L, Matos A. Depression in women with endometriosis with and without chronic pelvic pain. Acta Obstet Gynecol 2006;85:88-92.

21. Verit FF, Verit A, Yeni E. The prevalence of sexual dysfunction and associated risk factors in women with chronic pelvic pain: a cross-sectional study. Arch Gynecol Obstet 2006;274:297-302.

22. Kaya B, Unal S, Ozenli Y, Gursoy N, Tekiner S, Kafkasli A. Anxiety, depression and sexual dysfunction in women with chronic pelvic pain. Sex Relat Ther 2006;21:187-96.

23. Clemens JQ, Brown SO, Calhoun EA. Mental health diagnoses in patients with interstitial cystitis/painful bladder syndrome and chronic prostatis/chronic pelvic pain syndrome: a case/control study. J Urol 2008;180:1378-82.

24. Latthe P, Lathe M, Say L, Gülmezoglu M, Khan KS. WHO systematic review of prevalence of chronic pelvic pain: a neglected reproductive health morbidity. BMC Publ Health 2006;6:177.

25. Ghaly AF, Chien PF. Chronic pelvic pain: clinical dilemma or clinician's nightmare. Sex Transm Inf 2000;76:419-25.

26. Fox SD. Chronic pelvic pain in women. Med Health R I 2003;86:9-11. 
27. Fall M, Baranowski AP, Fowler CJ, Lepinard V, MaloneLee JG, Messelink EJ, et al. EAU guidelines on chronic pelvic pain. Eur Urol 2004;46:681-9.

28. Varma R, Gupta JK. Chronic pelvic pain: a practical approach. Women's Health Med 2005;2:5-12.

29. Cheong Y, Stones W. Investigations for chronic pelvic pain. Reviews in Gynaecological Practice 2005;5:227-36.

30. Ortiz DD. Chronic pelvic pain in women. Am Fam Physician 2008;77:1535-42.

31. Vincent K. Chronic pelvic pain in women. Postgrad Med J 2009;85:24-9.

32. Fletcher SG, Zimmern PE. Differential diagnosis of chronic pelvic pain in women: the urologist's approach. Nat Rev Urol 2009;6:557-62.

33. Khawaja M, Kaddour A, Zurayk H, Choueiry N, ElKak F. Symptoms of reproductive tract infections and mental distress among women in low-income urban neighborhoods of Beirut, Lebanon. J Womens Health 2009;18:1701-8.

34. Bachman GA, Rosen R, Arnold LD, Burd I, Rhoads GG, Leiblum SR, et al. Chronic vulvar and other gynecologic pain: prevalence and characteristics in a self-reported survey. J Reprod Med 2006;51:3-9.

35. Grace V, Zondervan K. Chronic pelvic pain women in New Zealand: comparative well-being comorbidity, and impact on work and other activities. Health Care Women Int 2006;27:585-99.

36. Pitts MK, Ferris JA, Smith AM, Shelley JM, Richters J. Prevalence and correlates of three types of pelvic pain in a nationally representative sample of Australian women. Med J Aust 2008;189:138-43.

37. Marszalek M, Wehrberger C, Temml C, Ponholzer A, Berger I, Madersbacher S. Chronic pelvic pain and lower urinary tract symptoms in both sexes: analysis of 2749 participants of an urban health screening project. Eur Urol 2009;55:499-508.

38. Hernández Ávila M, Garrido F, Salazar Martínez E. Sesgos en estudios epidemiológicos. Salud Pública Méx 2000;42:438-46.
39. Grimes DA, Schulz KF. Bias and causal associations in observational research. Lancet 2002;359:248-52.

40. Sica GT. Bias in research studies. Radiology 2006;238:780-9.

41. Asociación Colombiana para el Estudio del Dolor. Encuesta nacional del dolor, 2000. Visitado en 2010 Oct 25. Disponible en: www.dolor.org.co

42. Asociación Colombiana para el Estudio del Dolor. Segunda encuesta nacional del dolor, 2002. Visitado en 2010 Oct 25. Disponible en: www.dolor.org.co

43. Asociación Colombiana para el Estudio del Dolor. Tercer estudio nacional del dolor, 2004. Visitado en 2010 Oct 25. Disponible en: www.dolor.org.co

44. Asociación Colombiana para el Estudio del Dolor. Cuarto estudio nacional del dolor, 2008. Visitado en 2010 Oct 25. Disponible en: www.dolor.org.co

45. Asociación Colombiana para el Estudio del Dolor. Quinto estudio nacional del dolor, 2010. Visitado en 2010 Oct 25. Disponible en: www.dolor.org.co

46. Gómez PI. Dolor pélvico crónico en la mujer. En: Gómez PI, Hernández JJ (eds). Dolor en la mujer. Bogotá: Asociación Colombiana para Estudio del Dolor; 2008. p. 105-23.

47. Mueller ER, Kenton K, Rogers RG, Fenner DE. Are we missing an opportunity to teach future physicians about female pelvic floor disorders? Int Urogynecol J Pelvic Floor Dysfunction 2009;20:1413-5.

48. McGowan L, Escott D, Luker K, Creed F, ChewGraham C. Is chronic pelvic pain a comfortable diagnosis for primary care practitioners: a qualitative study. BMC Fam Pract 2010;11:7.

49. McGowan-Linda PA, Clark-Carter DD, Pitts MK. Chronic pelvic pain: a meta-analytic review. Psychol Health 1998;13:937-51.

50. Latthe P, Mignini L, Gray R, Hills R, Khan K. Factors predisposing women to chronic pelvic pain: a systematic review. BMJ 2006;332:749-55. 